\title{
La formación de educadores ambientales en México: avances y perspectivas
}

\section{A formação de educadores ambientais no México: progressos e perspectivas}

\section{Training of environmental educators in Mexico: progress and prospects}

\author{
Édgar J. González-Gaudiano ${ }^{1}$ \\ Miguel Ángel Arias-Ortega ${ }^{2}$
}

\begin{abstract}
RESUMEN
La formación de educadores ambientales fue planteada como un imperativo del campo desde los primeros años de su implantación en México. Si bien el desarrollo de programas de profesionalización en la forma de diplomados, especializaciones y maestrías ha sido continuo, también ha sido vulnerable a los cambios en las propias universidades de adscripción, así como a los de las políticas ambientales gubernamentales. El presente artículo da cuenta de esta historia. Se conceptualiza el problema, se resume el perfil actual que se observa y se apuntan algunas salidas que se han ido construyendo para fortalecer este campo. En este sentido, destaca especialmente el hecho de que diversas maestrías y doctorados en ciencias o en educación aceptan propuestas de investigación en educación ambiental. Con ello, la esfera de profesionalización se ha expandido más allá del circuito de los educadores ambientales, lo que ha favorecido el diálogo y los intercambios creativos y productivos con otras áreas de conocimiento y alrededor de diversas temáticas, entre ellas lo intercultural, la conservación, las ciencias, el cambio climático, la literatura y la poesía, entre otras, que en su conjunto ofrecen
\end{abstract}

\section{DOI: $10.1590 / 0104-4060.49136$}

1 Instituto de Investigaciones en Educación. Universidad Veracruzana. Xalapa, Veracruz, México. Campus Sur. Paseo 112, Lote 2, Sección 2A, Edificio B, $2^{\circ}$ piso, Col. Nuevo Xalapa, 91097, Xalapa, Ver.E-mail: edgagonzalez@uv.mx

2 Universidad Autónoma de la Ciudad de México. Del. Benito Juárez, Ciudad de México, México. San Lorenzo, 290, Col Del Valle, 03100, Del. Benito Juárez, Ciudad de México. E-mail: maao@servidor.unam.mx 
una perspectiva sobre los procesos de profesionalización de los educadores ambientales en México.

Palabras-clave: educación ambiental; profesionalización; desafíos de la formación.

\title{
RESUMO
}

A formação de educadores ambientais foi estabelecida como um imperativo do campo desde os primeiros anos da sua aplicação no México. Embora o desenvolvimento de programas de formação profissional sob a forma de diplomas, especializações e mestrados tenha sido um contínuo, também tem sido vulnerável a mudanças nas universidades de filiação, bem como nas políticas ambientais governamentais. Este artigo relata esta história. Para isso, o problema é conceituado, o perfil atual observado é resumido e algumas saídas, que foram construídas para fortalecer esse campo, são descritas. Neste sentido, especialmente destaca-se o fato de vários mestrados e doutorados em ciência ou educação aceitarem propostas de pesquisas em educação ambiental. Desta forma, a esfera da profissionalização se expandiu para além do circuito dos educadores ambientais, o que favoreceu o diálogo e o intercâmbio criativo e produtivo com outras áreas de conhecimento e em torno de diversas temáticas, entre elas a intercultural, a conservação, as ciências, as alterações climáticas, a literatura e a poesia, entre outras, que juntas oferecem uma perspectiva sobre o processo de profissionalização dos educadores ambientais no México.

Palavras-chave: educação ambiental; profissionalização; desafios da formação.

\begin{abstract}
The training of environmental educators was established as an imperative of the field since the early years of its implementation in Mexico. While the development of professional training programs in the form of diplomas, specialization and masters has been continuous, it has also been vulnerable to changes in the universities of affiliation, as well as in government environmental policies. This article reports on this story. For this purpose, the problem is conceptualized, the current profile is found and summarized and some routes that have been designed to strengthen this field are described. In this regard, it particularly highlights the fact that various masters and doctorates in science or education accept proposals to research for environmental education. In this way, the sphere of professionalization has expanded beyond the environmental educators' circuit, which has favored dialogue and creative and productive exchanges with other areas of education
\end{abstract}


such as intercultural education, conservation, science, climate change, literature and poetry, among others, which together offer a perspective on the process of professionalization of environmental educators in Mexico.

Keywords: environmental education; professionalization; training challenges.

\section{Sobre la profesionalización}

Para los fines del presente artículo partimos de las ideas de Macdonald (1997), quien menciona que el concepto de profesión (del latín profiteri, cuyo sentido original de profesar se vinculaba a las órdenes religiosas), se secularizó entre 1700 y 1800 absorbiendo a las profesiones liberales, tales como medicina, leyes, la milicia, educación y ciencias naturales. Alrededor del año 1900, este concepto comprendía los atributos y rasgos que separaban a las profesiones de otras ocupaciones, justificando prácticas discriminatorias hacia las diferencias de raza, género y clase. Los profesionales se representaban más por lo que no eran que por lo que eran, asumiéndose como expertos en un cierto campo de conocimiento vital y hermético (iniciático). En esta etapa, se formalizaron los procesos de certificación y se especificaron los códigos de ética. A mediados del siglo XX la profesionalización en función de atributos cambió por una caracterización con base en procesos.

En ese devenir la educación ha sido considerada como una profesión débil (dependiente y con autonomía limitada) por su relación con el Estado y por carecer de un conocimiento hermético, aunque con asociaciones profesionales fuertes. Si bien el debate sobre el profesional de la educación es constante, el del educador ambiental es imperativo y ha sido constante desde inicios de los años 90 del siglo pasado. La propia identidad del educador ambiental es aún difusa. Desde hace varias décadas ha habido una discusión en términos dicotómicos sobre si el educador ambiental es un especialista o un generalista. (GÓMEZ; RAMOS, 1989). Otros lo han descrito como un campo todavía en el útero (MACDONALD, 1997), donde converge toda suerte de perfiles, desde los expertos hasta los aficionados (amateurs). Desde nuestra perspectiva es preciso replantear algunos términos, puesto que coincidimos con Popkewitz en cuanto a que:

La pertenencia a las comunidades científicas supone participar de ciertas premisas y líneas de razonamiento que hacen válido el conocimiento. Cada 
área científica dispone de un conjunto particular de preguntas, métodos y procedimientos. Estos conjuntos proporcionan formas compartidas de "ver" el mundo, de trabajar y de comprobar los estudios de los demás. La formación de un individuo con vistas a su integración en una comunidad científica exige algo más que el aprendizaje de los contenidos de la disciplina correspondiente. Se aprende también a mirar, pensar y actuar en el mundo de cierta manera. (POPKEWITZ, 1988, p. 31).

Lo anterior es consistente con la teoría de campos sociales de Pierre Bourdieu (1990, p. 108-112), quien define a un campo como un espacio históricamente constituido con instituciones específicas, normas de funcionamiento propias y capital simbólico compartido, en el que se da una lucha permanente entre quienes detentan el poder y quienes aspiran a obtenerlo ${ }^{3}$. Por lo mismo, el conflicto es constitutivo de todo campo al coexistir perspectivas que rivalizan entre sí y ponen atención a problemas distintos ofreciendo respuestas diferentes, y donde lo constitutivo de dicho campo, es la tensión permanente por sedimentar y normalizar una postura frente a otra. El campo de la educación en general y de la educación ambiental en particular no es ajeno a esta condición en la que confluyen distintos intereses en pugna.

\section{La profesionalización en educación ambiental en México}

La discusión sobre el campo de la educación ambiental permite entender el marco en el que está inserta la posibilidad de la profesionalización. En este sentido, nos posicionamos en un concepto de profesionalización que no está adscrito necesariamente a la formación universitaria. Esto es, si bien admitimos la hegemonía de la institución escolar en el ámbito de la profesionalización, percibimos un horizonte de posibilidades más amplio que el reconocimiento expreso que una institución académica otorga para el ejercicio de una práctica profesional específica ${ }^{4}$. El criterio académico es válido para aquellos que egresan

3 Fernández Crispín (2013) abre una discusión sobre la formación de educadores ambientales desde la interculturalidad, las ciencias y las prácticas de intervención docente.

4 Gutiérrez y otros (1997, p. 6) mencionan que en el desarrollo profesional de los agentes o educadores ambientales suelen no abordarse "[...] las cuestiones específicas relativas al perfil profesional de los buenos educadores ambientales, ni tampoco a las instituciones formativas que debe ocuparse de estos temas. El contenido central de este debate se ha volcado en las estrategias 
de programas universitarios sobre educación ambiental, pero no para todos, e incluso ni siquiera para todos los propios egresados.

En México los programas académicos vinculados con la educación ambiental se iniciaron hace aproximadamente veinte años, primero mediante forma de diplomados ${ }^{5}$ luego de maestrías ${ }^{6}$. En este ámbito se ha observado desde el inicio una amplia pluralidad de enfoques y aproximaciones a la tarea formativa. En parte esto se debe a la composición del área disciplinaria promotora (biología, química, humanidades, etc.), la cual por sí misma genera sesgos formativos. Ello incluso sería conveniente si existiera algún acuerdo explícito por atender las numerosas y diferenciadas necesidades socio-profesionales del campo, por ejemplo que quienes se forman como educadores ambientales en programas adscritos al área de las ciencias, estuvieran abocados a poner en marcha programas de educación ambiental centrados en problemáticas afines a las disciplinas del área, como las que enfrentan quienes trabajan vinculados a programas de manejo de vida silvestre o en zonas naturales protegidas. El problema consiste en que esta pluralidad de perfiles formativos responde a los sesgos existentes del área de adscripción que imprime una orientación particular a cada propuesta curricular, lo que no siempre habilita para desempeñarse apropiadamente en un campo con necesidades y prácticas tan diversas.

En el contexto mexicano fueron los biólogos y los ecólogos quienes dieron las primeras voces de alarma sobre los problemas ambientales y quienes empezaron a instrumentar programas educativos tendientes a enfrentar el problema, de ahí que en su mayoría, las propuestas educativas que emergían se acompañaban de una primera unidad donde se abordaban los procesos biofísicos de la naturaleza: ecosistema, bioma, nicho ecológico, fotosíntesis, etc., sin ninguna vinculación con los aspectos sociales. El momento inicial de la oferta formativa se dio durante la década de los años 90, cuando de acuerdo con la base de datos del Centro de Educación y Capacitación para el Desarrollo Sustentable de la Secretaría de Medio Ambiente, Recursos Naturales y Pesca (CECADESU, 1997) se ofrecían en México nueve diplomados, dos especialidades y cinco maestrías. Cuatro de esos diplomados estaban vinculados institucionalmente con el área de

formativas, y muy especialmente, en las estrategias de autoformación protagonizadas por los agentes en activo".

5 Los diplomados en México son estudios de educación continua orientados a la actualización de profesionistas que ya se encuentran en el mercado laboral. Sus objetivos consisten fundamentalmente en dotarlos de nuevas habilidades, destrezas y conocimientos que no fueron cubiertos en su formación original o que han quedado desactualizados. Administrativamente se catalogan de manera distinta a estudios técnicos, profesionales y de posgrado.

6 El primer programa de maestría en educación ambiental fue ofertado por la Universidad Pedagógica Nacional. Unidad 095 Azcapotzalco, Ciudad de México, el 09 de marzo de 1992. 
Biología, tres con la química con orientación a ingeniería y dos con humanidades. La mayor parte de las maestrías y las dos especializaciones estaban vinculadas con la pedagogía y, específicamente, con la formación de maestros. La maestría que continúa ofreciendo la Universidad de Guadalajara es el único programa que ha operado bajo la modalidad de educación a distancia y aunque está vinculada institucionalmente con el área de Ciencias Biológicas y Agropecuarias, tiene un enfoque interdisciplinario ${ }^{7}$. Actualmente, las especializaciones están suspendidas y los diplomados no son permanentes. Sin embargo, el número de maestrías que se imparten en México ha aumentado y se han diversificado sus enfoques. Como se puede ver en el Cuadro 1, son 16 programas de maestría de las cuales 14 están vinculados al área educativa y de las Humanidades y cinco (31\%) concentradas en la zona metropolitana de la Ciudad de México. Sólo dos de estos programas se imparten en universidades privadas y la mitad de estas instituciones están orientadas a la formación profesional de profesores.

Una nueva situación que consideramos es la vía formativa de mayor potencial en este momento, al menos en México, es el hecho de que varias maestrías $\mathrm{y}$ doctorados en educación y en ciencias abren la oportunidad para presentar proyectos de tesis de intervención en educación ambiental. Algunos de ellos incluso tienen líneas de investigación u orientaciones curriculares definidas, como es el caso de la maestría y el doctorado en investigación educativa de la Universidad Veracruzana, o la maestría en intervención e innovación para el desarrollo educativo de la Universidad Pedagógica en el estado de Hidalgo.

El nuevo espacio formativo está asociado al de la aparición de cuerpos académicos y grupos de investigación en educación ambiental en diversas instituciones de educación superior. En el reporte de González-Gaudiano y Arias (2015) se registran 25, once de los cuales “[...] están ubicados en el área de las ciencias naturales, ocho en disciplinas sociales y humanidades, cuatro en el área tecnológica e ingenierías y solo dos en el área de diseño y artes". (GONZÁLEZ-GAUDIANO; ARIAS, 2015, p. 63).

Asimismo, en el mismo reporte (GONZÁLEZ-GAUDIANO; ARIAS, 2015 , p. 62 y p. 82-85) puede consultarse la situación relativa a la producción de tesis de licenciatura, maestría y doctorado, que fue de 379 en el periodo 20022011, lo que representó un incremento de $19.9 \%$ respecto al decenio anterior. De estos, $53 \%$ correspondió al nivel de maestría y 5\% de doctorado. En relación con este último dato, el reporte mostró que en el periodo de referencia se presentaron 19 tesis doctorales en el campo de la educación ambiental, lo cual representa

7 La Maestría en Educación Ambiental a Distancia de la Universidad de Guadalajara fue aprobada en el mes de octubre de 1994 y es en el mes de septiembre de 1995 cuando inician de manera formal los cursos académicos. 
CUADRO 1 - PROGRAMAS DE MAESTRÍA EN EDUCACIÓN AMBIENTAL EN MÉXICO

\begin{tabular}{|c|c|c|c|c|}
\hline $\mathbf{N}^{\circ}$ & Nombre del Programa & $\begin{array}{l}\text { Universidad / Área de } \\
\text { Adscripción }\end{array}$ & Modalidad & $\begin{array}{l}\text { Estado / } \\
\text { Región }\end{array}$ \\
\hline 1 & $\begin{array}{l}\text { Maestría Educación } \\
\text { Ambiental para el } \\
\text { Desarrollo Sustentable }\end{array}$ & $\begin{array}{l}\text { Universidad de Quintana Roo / } \\
\text { Ingeniería y Ciencias Aplicadas }\end{array}$ & Escolarizada & $\begin{array}{l}\text { Quintana Roo / } \\
\text { Sureste }\end{array}$ \\
\hline 2 & $\begin{array}{l}\text { Maestría en Educación } \\
\text { Ambiental (EA) }\end{array}$ & $\begin{array}{l}\text { Universidad Pedagógica Nacional, } \\
\text { Unidad 095, Azcapotzalco / } \\
\text { (UPN). Educación y Humanidades }\end{array}$ & Escolarizada & $\begin{array}{l}\text { Ciudad de } \\
\text { México / } \\
\text { Centro }\end{array}$ \\
\hline 3 & $\begin{array}{l}\text { Maestría en Educación } \\
\text { con campo en Educación } \\
\text { Ambiental }\end{array}$ & $\begin{array}{l}\text { UPN. Unidad 251, Culiacán / } \\
\text { Educación y Humanidades }\end{array}$ & Escolarizada & $\begin{array}{l}\text { Sinaloa / } \\
\text { Noroeste }\end{array}$ \\
\hline 4 & $\begin{array}{l}\text { Maestría en Educación } \\
\text { con campo en Educación } \\
\text { Ambiental }\end{array}$ & $\begin{array}{l}\text { UPN. Unidad 252, Mazatlán / } \\
\text { Educación y Humanidades }\end{array}$ & Escolarizada & $\begin{array}{l}\text { Sinaloa / } \\
\text { Noroeste }\end{array}$ \\
\hline 5 & $\begin{array}{l}\text { Maestría en Educación } \\
\text { Ambiental }\end{array}$ & $\begin{array}{l}\text { UPN del Estado de Sinaloa / } \\
\text { Educación y Humanidades }\end{array}$ & Escolarizada & $\begin{array}{l}\text { Sinaloa / } \\
\text { Noroeste }\end{array}$ \\
\hline 6 & $\begin{array}{l}\text { Maestría en Ciencias de } \\
\text { la Educación Ambiental }\end{array}$ & $\begin{array}{l}\text { Universidad Juárez Autónoma } \\
\text { de Tabasco / Educación y } \\
\text { Humanidades }\end{array}$ & Escolarizada & $\begin{array}{l}\text { Tabasco / } \\
\text { Sureste }\end{array}$ \\
\hline 7 & $\begin{array}{l}\text { Maestría en Educación } \\
\text { Ambiental y Desarrollo } \\
\text { Regional }\end{array}$ & $\begin{array}{l}\text { Centro Regional de Educación } \\
\text { Superior "Paulo Freire"/ } \\
\text { Educación y Humanidades }\end{array}$ & Escolarizada & $\begin{array}{l}\text { Veracruz / } \\
\text { Sureste }\end{array}$ \\
\hline 8 & $\begin{array}{l}\text { Maestría en Educación } \\
\text { (Educación Ambiental) }\end{array}$ & $\begin{array}{l}\text { UPN. Unidad 021, Mexicali / } \\
\text { Educación y Humanidades }\end{array}$ & Escolarizada & $\begin{array}{l}\text { Baja California } \\
\text { Norte/Noroeste }\end{array}$ \\
\hline 9 & $\begin{array}{l}\text { Maestría en Educación } \\
\text { Ambiental }\end{array}$ & $\begin{array}{l}\text { Instituto de Estudios de Posgrado/ } \\
\text { Educación y Humanidades }\end{array}$ & Escolarizada & Chiapas / Sur \\
\hline 10 & $\begin{array}{l}\text { Maestría en Educación } \\
\text { Ambiental para la } \\
\text { Sustentabilidad } \\
\end{array}$ & $\begin{array}{l}\text { Instituto de Estudios de Posgrado/ } \\
\text { Ciencias Exactas y Naturales }\end{array}$ & Escolarizada & Chiapas / Sur \\
\hline 11 & $\begin{array}{l}\text { Maestría en Educación } \\
\text { Ambiental }\end{array}$ & $\begin{array}{l}\text { UPN, Unidad 092, Ajusco / } \\
\text { Educación y Humanidades }\end{array}$ & Escolarizada & $\begin{array}{l}\text { Ciudad de } \\
\text { México / } \\
\text { Centro } \\
\end{array}$ \\
\hline 12 & $\begin{array}{l}\text { Maestría en Educación } \\
\text { con campo en Educación } \\
\text { Ambiental }\end{array}$ & $\begin{array}{l}\text { UPN, Unidad 092, Ajusco / } \\
\text { Educación y Humanidades }\end{array}$ & Escolarizada & $\begin{array}{l}\text { Ciudad de } \\
\text { México / } \\
\text { Centro } \\
\end{array}$ \\
\hline 13 & $\begin{array}{l}\text { Maestría en Educación } \\
\text { Ambiental }\end{array}$ & $\begin{array}{l}\text { Universidad Autónoma de la } \\
\text { Ciudad de México / Educación y } \\
\text { Humanidades }\end{array}$ & Escolarizada & $\begin{array}{l}\text { Ciudad de } \\
\text { México / } \\
\text { Centro } \\
\end{array}$ \\
\hline 14 & $\begin{array}{l}\text { Maestría en Educación } \\
\text { Ambiental }\end{array}$ & $\begin{array}{l}\text { Universidad de Guadalajara / } \\
\text { Educación y Humanidades }\end{array}$ & A distancia & $\begin{array}{l}\text { Jalisco / } \\
\text { Occidente }\end{array}$ \\
\hline 15 & $\begin{array}{l}\text { Maestría en Educación } \\
\text { Ambiental }\end{array}$ & $\begin{array}{l}\text { Universidad Intercultural Indígena } \\
\text { de Michoacán / Educación y } \\
\text { Humanidades }\end{array}$ & Escolarizada & $\begin{array}{l}\text { Michoacán / } \\
\text { Occidente }\end{array}$ \\
\hline 16 & $\begin{array}{l}\text { Maestría en Educación y } \\
\text { Comunicación Ambiental }\end{array}$ & $\begin{array}{l}\text { Universidad de la Comunicación / } \\
\text { Educación y Humanidades }\end{array}$ & Escolarizada & $\begin{array}{l}\text { Ciudad de } \\
\text { México/Centro }\end{array}$ \\
\hline
\end{tabular}

FUENTE: Los autores (2016). 
un hallazgo altamente valorado, toda vez que el hecho permite vislumbrar otras formas de acercamiento, vinculación y diálogo de la educación ambiental con otras áreas de conocimiento que, sin duda, serán de enorme enriquecimiento para los educadores ambientales y para el campo mismo, y que se configura en una trayectoria que debe ser fomentada en los años por venir.

Algunas instituciones académicas ofrecen también programas formativos cortos no reconocidos como estudios de posgrado, sino como de educación continua. El mismo reporte citado arriba registra 22 de estos programas, de los cuales 19 son diplomados (85\%); $27 \%$ tiene una modalidad virtual y $9 \%$ semi-presencial. (GONZÁLEZ-GAUDIANO; ARIAS, 2015, p. 94).

Complementariamente existen experiencias de organizaciones de la sociedad civil (OSC) que han impulsado la formación de sus cuadros y de personas interesadas en el tema, bajo modalidades prácticas vinculadas con la resolución de problemas sociales específicos, es el caso, por ejemplo, del Grupo Ecológico Sierra Gorda en el estado de Querétaro, México. De ahí que a menudo su enfoque central no es el ambiente, sino la satisfacción de necesidades básicas. No obstante, algunos de los proyectos de educación ambiental mejor consolidados se encuentran amalgamados con programas de desarrollo comunitario. Estas experiencias se llevan a cabo con mayor frecuencia en áreas rurales y a veces ni siquiera se plantean como proyectos de educación ambiental, puesto que lo educativo se encuentra articulado con otros elementos culturales, productivos y sociales que configuran un proyecto de mayor alcance.

En relación con la participación de las organizaciones de la sociedad civil en procesos de formación de educadores ambientales, podemos advertir que ha sido una actividad que, aunque se han hecho presente desde la década de los años noventa, no ha sido un proceso permanente ni ha mostrado un incremento en el número de propuestas formativas, en la medida que muchas de ellas han estado más enfocadas a subsanar sus necesidades económicas de financiamiento que a promover un programa académico consistente y con amplias proyecciones para formar y actualizar a educadores ambientales en el país. No obstante, en la actualidad se vislumbran propuestas formativas emanadas de este tipo de organizaciones, a las cuales debemos estar atentos en su desarrollo dentro del campo de la educación ambiental en el contexto mexicano.

De igual manera, algunos centros de educación ambiental, así como equipamientos que ofrecen diversos servicios culturales tales como museos, zoológicos y jardines botánicos cuentan también con estrategias formativas del personal que reclutan. La preparación consiste en un entrenamiento usualmente corto a fin de que se encuentren en condiciones de conducir los distintos itinerarios que forman parte de sus programas, principalmente el desarrollo de actividades al aire libre y, comúnmente dirigidas a grupos escolares. Sin embargo, en estos 
casos suelen ser estrategias más informativas que formativas que soslayan las causas de problemas e incluso a menudo sus consecuencias directas e indirectas en la vida de las personas, simplemente ni se mencionan.

\section{Los desafíos que enfrentamos}

La Agenda para la educación de personas adultas, de Joaquín Esteva y Javier Reyes (1997), plantea que, desde una perspectiva ambiental:

La educación entre personas adultas debe enfatizar la relación indisoluble entre desarrollo y ambiente, considerando a este como un espacio de posibilidades y satisfacciones... [Asimismo] debe propiciar la formación colectiva de sujetos autónomos que analicen críticamente la naturalidad de su realidad y puedan poner en tela de juicio la normativa instituida. Esto implicaría a su vez crear una institucionalidad cuya interiorización no limite sino amplíe la capacidad de llegar a ser sujetos críticos de esa misma institucionalidad. (ESTEVA; REYES, 1997, p. 39).

Por lo anterior, la formación práctica que puede obtenerse mediante la actividad directa ofrece un amplio arco de posibilidades. Sin embargo, debemos estar atentos a la distorsión que el empirismo puede inducir dentro de la experiencia. Toda actividad práctica requiere de momentos de evaluación crítica, de un análisis honesto de los resultados, de una reflexión hacia adelante para poder incorporar oportunamente las medidas necesarias en función del contexto y los sujetos a los que se dirigen las acciones educativas. La autocomplacencia en la que incurrimos cuando trabajamos sobre la base de tareas que parecen exitosas en el arranque - situación frecuente en la educación ambiental - suele producir espejismos sobre sus posibilidades en el mediano y largo plazos. Los espejismos se pagan caros en el trabajo social y sus consecuencias, en lo general provocan que las personas no manifiesten una participación social comprometida, respecto a lo ambiental.

Ambas modalidades, la académica y la práctica, operan como estrategias de profesionalización en el sentido planteado anteriormente. En otras palabras, si la educación ambiental tiene como misión contribuir a la formación de nuevos conocimientos, valores, habilidades y competencias sobre la relación de las 
sociedades consigo mismas y con el ambiente, entonces un profesional de la educación ambiental puede verse como alguien que desarrolla dicha práctica social en el marco de un proyecto pedagógico posible, cualquier que este sea, pero con un sentido definido, manifiesto o no. Nuestro campo se compone de una multiplicidad de prácticas, dispersas y puntuales unas, organizadas y dirigidas otras. Su re-conocimiento es necesario para valorar sus distintas contribuciones y alcances, así como para poder estar en mejores condiciones de producción en la trama de discursos que nos constituyen y que sobre-determinan nuestras diferentes formas de percibir y actuar en los asuntos que nos competen en todos los ámbitos de la vida cotidiana, ya sea en lo individual como en lo colectivo.

Gee, Hull y Colin Lankshear (1996, p. 10), siguiendo a Pierre Bourdieu y Michel Foucault, sostienen que un Discurso crea posiciones sociales (o perspectivas) de aquellos que son "convocados" a hablar, escuchar, actuar, leer y escribir, pensar, sentir, creer y valorar en ciertas formas características, históricamente reconocibles, en combinación con su propio estilo y creatividad individual. Señalan también que cada Discurso contrae complejas relaciones de complicidad, tensión y oposición con otros Discursos ${ }^{8}$. Si aceptamos que la educación ambiental está investida por distintos tipos de Discursos que constituyen diferentes clases de educadores ambientales, con variadas perspectivas y compromisos, ¿no será preciso identificar los principales Discursos que transitan por el campo de la educación ambiental, así como las tensiones y conflictos entre ellos, para así poder pensar en las condiciones de posibilidad que darán sentidos definidos a los procesos de formación de educadores ambientales? Si no tomamos esto en cuenta corremos un doble riesgo. Primero, porque se podría asumir que el único discurso profesionalizante es el discurso académico de la educación ambiental y desde ahí este puede auto-investirse con el carácter de legítimo y excluyente de discursos construidos por prácticas no académicas, que con demasiada frecuencia suelen descalificarse por ser producto, únicamente de la experiencia de los individuos. Segundo, porque podríamos creer que se puede construir un discurso de la educación ambiental apropiado para la realidad de nuestros pueblos sin reconocer sus verdaderos impactos en nuestros cuerpos y mentes.

Es difícil pensar en un proyecto de profesionalización de la educación ambiental sin un ideario pedagógico ${ }^{9}$ que nos posibilite reactivar la reflexión y práctica educativa, abriendo alternativas discursivas que des-sedimenten el Discurso de la educación ambiental de los significados fijos, de los metarrelatos omnicomprensivos sobre la naturaleza y de aquellas representaciones que apelan

8 Los autores utilizan "Discurso" con 'D' mayúscula para distinguirlo de "discurso", que significa "una extensión de lenguaje hablado o escrito" o "lenguaje en uso".

9 Se recupera el concepto de ideario tal como lo trabaja Isabel Carvalho (2001, p. 17). 
a una relación imposiblemente idílica entre la sociedad y el ambiente. Para pensar en ese ideario pedagógico estamos convencidos de que tenemos que pensar en un sistema abierto que dé cabida a los diferentes perfiles que requieren los distintos escenarios de intervención pedagógica, aunque con una cierta unidad discursiva que permita articular las diversas actividades en torno a un proyecto político en continua construcción y revisión. Nos hemos esforzado por desarrollar una pedagogía ambiental capaz de abrir nuevos cauces de participación social con base en la comprensión y asunción de los derechos y obligaciones de cada quien, del cuestionamiento permanente y del involucramiento constante en los asuntos de su vida cotidiana. De la misma manera, debemos sentar las bases para construir una política ambiental que redefina el rol a desempeñar por los procesos pedagógicos en la gestión ambiental más allá de una función instrumental y de la declaración de buenas intenciones en acuerdos que se convierten en meras referencias documentales. Se trata de un imaginario pedagógico que no deslinde la lucha por la defensa de la naturaleza, de la lucha por los derechos de la gente y por su dignidad y libertad posible. De toda la gente. Aún a sabiendas de que la desigualdad social es un problema con diferentes rostros y en múltiples dimensiones, sí podemos contribuir de distintas maneras a modificar los términos y planos de los conflictos y la condición social, desde la educación ambiental.

Ciertamente hemos avanzado en el camino de la profesionalización de la educación ambiental, aunque hemos sido autoindulgentes al juzgar los estadios de consolidación del propio campo. Si bien hemos trascendido la concepción pedagógica cuyo foco era el estudio ecológico de la naturaleza, y ahora enfrentamos una política internacional que promueve una concepción ligada al desarrollo sustentable, no estamos en condiciones de afirmar que a lo largo de estas cuatro décadas nuestro tránsito haya sido tan lineal y armónico como lo han descrito algunos reportes. (GONZÁLEZ MUÑOZ, 1996; STERLING; GEOFF, 1992). Tampoco podemos admitir que los logros obtenidos en cada década constituyen una fase superior a la anterior. (NOVO, 1986). Es claro que el Discurso crítico y un tanto subversivo de la educación ambiental que se formuló en Chosica (1976) y Bogotá (1985) se encuentra fuertemente tensionado por la capacidad que el sistema neoliberal tiene para metabolizar la crítica social.

La profesionalización debe verse como un componente estrechamente ligado con los procesos de investigación en el campo de la educación ambiental ${ }^{10}$.

10 Lo anterior no solo en relación con las experiencias de formación profesional ligadas a proyectos de investigación (PAPADIMITRIOU, 1995, p. 85-97), sino por la necesidad de personal especializado con el enfoque académico requerido para fortalecer esta vertiente del campo que ha estado desatendida en la región y particularmente en América Latina y el Caribe. Una discusión interesante sobre la situación de la investigación en el campo de la educación ambiental se encuentra en Mrazek (1996). 
La importancia de impulsar la investigación es un tópico en el que existe una generalizada coincidencia en la región y que se entiende como "[...] el conjunto de acciones sistemáticas y deliberadas que llevan a la formulación, diseño y producción de nuevos valores, teorías, modelos, sistemas, medios, evaluaciones, procedimientos y pautas de conducta dentro de los procesos educativos". (LATAPÍ, 2009, p. 199). Esto sobre todo ante la ausencia prácticamente total de referentes apropiados acordes con las características socioculturales y económicas de las poblaciones a las que se dirigen los proyectos educativos. En pocas palabras, sin investigación no hay campo y sin campo no hay posibilidades de profesionalizar $^{11}$.

Lo anterior quiere decir que existe una fuerte necesidad de impulsar la investigación en la formación de los educadores ambientales, como condición sine que non para visibilizar el campo de la educación ambiental en el conjunto amplio de las políticas educativas y ambientales. La investigación aplicada que se desarrolla como parte de las tesis profesionales y de posgrado ha sido importante pero insuficiente. Se requieren investigaciones de mayor alcance y profundidad sobre las causas sociales que determinan problemas ambientales, así como de los factores que restringen el cambio social en ese aspecto. En este marco, la ampliación de los espacios de investigación en educación ambiental y el fortalecimiento de los procesos de formación empírica deben ser dos de las acciones posibles, a fin de configurar espacios de diálogo, intercambio y debate que nos habiliten para plantear nuevas preguntas y construir distintas respuestas a los enormes desafíos que enfrentamos en el campo de lo ambiental. La historia es contundente, lo ofrecido hasta el momento ha sido insuficiente y limitado.

La profesionalización de los educadores ambientales puede convertirse en una tarea con más transcendencia social y política de lo que reconocemos en primera instancia. Los desafíos no son sencillos. Se trata de impulsar un proceso de profesionalización en un contexto donde lo profesionalizante se caracteriza por una impronta académica que se ha impuesto a otras formas de adquirir habilidades, conocimientos y valores. Impronta donde los acuerdos sociales se desvanecen para dar paso a visiones excluyentes mediante un conjunto de prácticas monopólicas impuestas. (NIXON; RANSON, 1997). La aparición de maestrías y programas de formación de profesores en el campo de la educación ambiental en la región ha aportado un nuevo aliento, pese a que las políticas ambientales en su conjunto y la educación ambiental entre ellas han perdido aceleradamente peso relativo desde el tope alcanzado en 1992.

11 El debate sobre la conformación de un programa de doctorado en educación ambiental será uno de los tópicos en los que los educadores mexicanos tendremos necesariamente que promover, con miras a fortalecer los espacios de profesionalización de éste campo de conocimientos. 
La profesionalización es un reto, pero también una oportunidad para poder estar en condiciones de construir los discursos y prácticas que permitan generar procesos de participación social e institucional con una clara conciencia y compromiso por un futuro mejor para todos.

\section{REFERENCIAS}

ARIAS ORTEGA, M. Á. Educación ambiental: crónica de un proceso de formación. México: Universidad Autónoma de la Ciudad de México-Newton-Edición y Tecnología Educativa, 2016.

BOURDIEU, P. Sociología y cultura. México: Grijalbo, 1990.

CARVALHO, I. C. de M. A invenção ecológica. Narrativas e trajetórias da educação ambiental no Brasil. Porto Alegre: Universidades/UFRGS, 2001.

CECADESU. Directorio de Programas Académicos de Instituciones Nacionales de Educación Superior en Medio Ambiente, Recursos Naturales y Pesca. México: ANUIES-SEMARNAP, 1997.

ESTEVA, J.; REYES J. (Ed.). Contribuciones educativas para sociedades sustentables. Pátzcuaro: México, CESE, 1997.

FERNÁNDEZ CRISPÍN, A. La educación ambiental en México. Definir el campus y emprender el habitus. México: Secretaría de Medio Ambiente y Recursos Naturales-Benemérita Universidad Autónoma de Puebla, 2013.

GEE, J. M.; HULL, G.; LANKSHEAR, C. The new work order. Behind the language of the new capitalism. Boulder: Westview Press, 1996.

GÓMEZ, G. J.; RAMOS, A. R. M. Bases ecológicas de la educación ambiental. In: SOSA, N. (Coord.). Educación ambiental. Sujeto, entorno y sistema. Salamanca: Amarú, 1989.

GONZÁLEZ-GAUDIANO, E.; ARIAS-ORTEGA, M. A. La investigación en educación ambiental para la sustentabilidad en México 2002-2011. México: ANUIES-COMIE, 2015.

GONZÁLEZ MUÑOZ, M. C. Principales tendencias y modelos de la educación ambiental en el sistema escolar. Revista Iberoamericana de Educación, n. 11, p. 13-71, Mayo-Agosto 1996.

GUTIÉRREZ, J.; PERALES, J.; BENAYAS, J.; CALVO, S. (Ed.). Lineas de investigación en educación ambiental. Granada: Junta de Andalucía-Universidad de Granada, 1997.

LATAPÍ, S. P. Finaleprestissimo. Pensamiento, vivencias y testimonios. México: Fondo de Cultura Económica, 2009. 
MACDONALD, M. Professionalization and environmental education: is public passion too risky for business?. Canadian Journal for Environmental Education, v. 2, p. 58-85, 1997.

MRAZEK, R. (Ed.). Paradigmas alternativos de investigación en educación ambiental. Guadalajara: Universidad de Guadalajara-NAAEE-Semarnap, 1996.

NIXON, J.; RANSON, S. Teorising 'agreement': the moral bases of the emergent professionalism within the 'new' management of education. Discourse Studies in the Cultural Politics of Education, v. 18, n. 2, p. 197-214, 1997.

NOVO, M. Educación y medio ambiente. Madrid: UNED, 1986.

PAPADIMITRIOU, V. Professional development of in-service primary teachers in environmental education: an action research approach. Environmental Education Research, v. 1 , n. 1, p. $85-97,1995$.

POPKEWITZ, T. S. Paradigmas e ideología en investigación educativa. Las funciones sociales del intelectual. Madrid: Mondadori, 1998.

STERLING, S.; COOPER, G. In touch. Environmental education for Europe. WWF UK, 1992.

Texto recibido en 03 de octubre de 2016. Texto aprobado en 04 de noviembre de 2016. 